\title{
Analysis of MYB expression and MYB-NFIB gene fusions in adenoid cystic carcinoma and other salivary neoplasms
}

\author{
Louis B Brill $\mathrm{II}^{1,4}$, William A Kanner ${ }^{1,4}$, André Fehr ${ }^{2,4}$, Ywonne Andrén ${ }^{2}$, \\ Christopher A Moskaluk ${ }^{1}$, Thomas Löning ${ }^{3}$, Göran Stenman ${ }^{2}$ and Henry F Frierson Jr ${ }^{1}$ \\ ${ }^{1}$ Department of Pathology, University of Virginia Health System, Charlottesville, VA, USA; \\ ${ }^{2}$ Cancer Center Sahlgrenska, Department of Pathology, The Sahlgrenska Academy at University of \\ Gothenburg, Gothenburg, Sweden and ${ }^{3}$ Institut für Pathologie, Albertinen-Krankenhaus, Hamburg, Germany
}

\begin{abstract}
Recent studies have shown that the recurrent $t(6 ; 9)(q 22-23 ; p 23-24)$ translocation in adenoid cystic carcinoma results in a novel fusion of the MYB proto-oncogene with the transcription factor gene NFIB. To determine the frequency of this finding, we used RT-PCR assays of the MYB and MYB-NFIB fusion transcripts, and immunohistochemistry for the MYB protein, to study adenoid cystic carcinomas and other epithelial tumors of the salivary glands, and head and neck region. MYB-NFIB fusion transcript was detected in 25 of 29 (86\%) frozen adenoid cystic carcinoma tumor samples, and in 14 of $32(44 \%)$ formalin-fixed paraffin-embedded adenoid cystic carcinoma tumor specimens. In contrast, the MYB-NFIB fusion was not expressed in non-adenoid cystic carcinoma neoplasms of the head and neck, confirming the high specificity of the MYB-NFIB fusion. Adenoid cystic carcinomas from various anatomic sites, including salivary gland, sinonasal cavity, tracheobronchial tree, larynx, breast, and vulva were repeatedly fusion-positive, indicating that adenoid cystic carcinomas located in different anatomic sites not only have important morphologic features in common, but also probably evolve through activation of the same molecular pathways. Studies of the expression of MYB revealed that $89 \%$ of the tumors, including both fusion-positive and fusion-negative cases, overexpressed MYB RNA. Similarly, $82 \%$ of adenoid cystic carcinomas stained positive for MYB protein, compared with $14 \%$ of non-adenoid cystic carcinoma neoplasms, indicating that MYB immunostaining may be useful for the diagnosis of adenoid cystic carcinoma, but that neoplasms sometimes in the differential diagnosis are also labeled. The latter are, however, fusion-negative. In summary, our studies show that MYB activation through gene fusion or other mechanisms is a major oncogenic event in adenoid cystic carcinoma occurring at various anatomic sites. In addition to being a diagnostically useful biomarker for adenoid cystic carcinoma, MYB and its downstream effectors are also novel potential therapeutic targets.

Modern Pathology (2011) 24, 1169-1176; doi:10.1038/modpathol.2011.86; published online 13 May 2011
\end{abstract}

Keywords: adenoid cystic carcinoma; gene fusion; immunohistochemistry; $M Y B ; N F I B$; salivary gland

Adenoid cystic carcinoma is one of the most common malignant salivary gland neoplasms, yet its signature oncogenic event was not defined until recently. ${ }^{1,2}$ Adenoid cystic carcinoma usually arises in the major and minor salivary glands, but also may develop in a wide range of other locations including

Correspondence: Dr HF Frierson Jr, MD, University of Virginia Health System, Department of Pathology, PO Box 800214, 1215 Lee Street, Office 3036, Charlottesville, VA 22908-0214, USA.

E-mail: hff@virginia.edu

${ }^{4}$ These authors contributed equally to this work.

Received 9 March 2011; revised and accepted 5 April 2011; published online 13 May 2011 the sinonasal tract, tracheobronchial tree, breast, vulva, and skin. ${ }^{3,4}$ In adenoid cystic carcinoma of salivary glands, there is no sex predilection, and the age distribution is wide. The tumors of the head and neck have a proclivity for perineural invasion, leading to a common clinical complaint of facial pain. Although typically slow growing, adenoid cystic carcinomas frequently metastasize and there is poor long-term survival. ${ }^{3-6}$ The histopathologic differential diagnosis for adenoid cystic carcinoma arising in the head and neck includes pleomorphic adenoma, monomorphic adenoma, polymorphous low-grade adenocarcinoma, and basaloid squamous cell carcinoma, among others. 
By histopathology and immunohistochemistry, the neoplastic cells of adenoid cystic carcinoma show both myoepithelial and ductal differentiation, with positivity for $\mathrm{p} 63, \mathrm{~S} 100$ protein, smooth muscle actin, and cytokeratins. ${ }^{7}$ Typically, the more peripheral cells of tumor nests and glands show myoepithelial differentiation, and there is abundant production of extracellular matrix and basement membrane components, especially in the cribriform pattern. Adenoid cystic carcinoma is also usually positive for KIT, which is most frequently absent in neoplasms in the differential diagnosis. ${ }^{8,9}$

Cytogenetically, adenoid cystic carcinoma is characterized by a tumor-type specific $t(6 ; 9)$ (q22-23;p23-24) translocation found as the sole anomaly in a subgroup of tumors. ${ }^{10-13}$ Recently, we showed that this translocation generates a fusion of the $M Y B$ proto-oncogene to the transcription factor gene $N F I B .{ }^{1}$ In the resulting $M Y B-N F I B$ fusion oncogene, which is highly overexpressed in adenoid cystic carcinoma, the $3^{\prime}$ part of $M Y B$, including several target sites for negatively regulating microRNAs, is replaced by the last coding exon(s) of NFIB. The predicted MYB-NFIB fusion protein retains the DNA-binding and transactivation domains of wild type MYB, and is therefore expected to activate MYB target genes. ${ }^{1,2}$ In our original study, we found the $M Y B-N F I B$ fusion in six of six adenoid cystic carcinomas with $t(6 ; 9)$ translocations, and in an additional five cases from which cytogenetic information was not available, suggesting that the fusion may be a useful biomarker for adenoid cystic carcinoma, and that deregulation of $M Y B$ along with its target genes are key oncogenic events in adenoid cystic carcinoma. ${ }^{1}$ Two subsequent studies found the $M Y B-N F I B$ fusion or $M Y B$-translocations in about one-third and two-thirds, respectively, of salivary adenoid cystic carcinomas. ${ }^{14,15}$ In contrast, non-adenoid cystic carcinoma salivary neoplasms were negative for the $M Y B-N F I B$ fusion. Interestingly, Mitani et $a l^{14}$ noted increased MYB expression in 17 of 20 adenoid cystic carcinomas with the $M Y B-N F I B$ fusion, but also in 14 of 20 fusionnegative adenoid cystic carcinomas. Taken together, these initial studies clearly demonstrate that $M Y B$ activation is a hallmark of adenoid cystic carcinoma, but that the true frequency of the MYB-NFIB fusion is still unclear. In order to shed additional light on this issue, we have now performed a combined molecular and immunohistochemical study of adenoid cystic carcinomas and non-adenoid cystic carcinoma salivary neoplasms with the following aims: to study the frequency of the MYB-NFIB fusion in both formalin-fixed paraffin embedded (FFPE) and frozen adenoid cystic carcinoma tumors derived from various anatomic sites; to study the expression of $M Y B$ by quantitative real-time PCR (qPCR) in fusion-positive and fusion-negative adenoid cystic carcinomas; and to study the expression of MYB protein by immunohistochemistry, and determine the value of a commercially available
anti-MYB antibody in the histopathologic differential diagnosis.

\section{Materials and methods}

\section{Tumor Material}

We reviewed the Surgical Pathology archives and those of the Adenoid Cystic Carcinoma Tumor Registry at the University of Virginia for adenoid cystic carcinoma from all anatomic sites, and for other salivary neoplasms. FFPE tumor material from 68 cases of adenoid cystic carcinoma, and 113 nonadenoid cystic carcinoma neoplasms of the head and neck was selected for molecular and/or immunohistochemical analyses (Table 1). Of the 68 adenoid cystic carcinomas, clinical information was available for all but three of the patients. A total of 53 tumors were from the aerodigestive tract; of these, 15 were located in the minor salivary glands, 13 in the parotid gland, nine in the trachea/ bronchus, eight in the sinonasal cavity, four in the submandibular gland, and four in the larynx. Adenoid cystic carcinomas at other anatomic sites included four that arose in the breast, three in the vulva, two in the lacrimal gland, and four were metastases. In all, 20 adenoid cystic carcinomas arose in men, whereas 45 were from women. The age range was $23-77$ years.

In addition, we had access to FFPE tumor material for RT-PCR analysis from eight adenoid cystic carcinomas, of which two were located in the major salivary glands, three in the trachea/bronchus, and one each in the larynx, breast, and vulva.

Moreover, fresh frozen tumor material was available for RT-PCR and qPCR analysis from 29 cases of adenoid cystic carcinoma, of which 23 were located

Table 1 Summary of immunohistochemical staining for MYB in adenoid cystic carcinoma of various anatomic sites, as well as other selected neoplasms of the head and neck

\begin{tabular}{lcc}
\hline Diagnosis & $\begin{array}{c}\text { MYB IHC } \\
(-)\end{array}$ & $\begin{array}{c}\text { MYB IHC } \\
(+)\end{array}$ \\
\hline Adenoid cystic carcinoma & 12 & 56 \\
& 97 & 16 \\
Non-adenoid cystic carcinoma & 15 & 1 \\
Acinic cell carcinoma & 1 & 4 \\
Basaloid squamous cell carcinoma & 3 & 1 \\
Basal cell adenoma & 2 & 1 \\
Basal cell adenocarcinoma & 3 & 0 \\
Carcinoma ex pleomorphic adenoma & 2 & 1 \\
Epithelial-myoepithelial carcinoma & 8 & 1 \\
Mucoepidermoid carcinoma & 3 & 1 \\
Monomorphic adenoma & 12 & 1 \\
Pleomorphic adenoma & 13 & 2 \\
Polymorphous low-grade & & \\
adenocarcinoma & 0 & 1 \\
Salivary adenoma, NOS & 1 & 0 \\
Salivary adenocarcinoma, NOS & 16 & 2 \\
Salivary duct carcinoma & 18 & 0 \\
Warthin tumor & &
\end{tabular}

Abbreviation: NOS, not otherwise specified. 
in the major or minor salivary glands, three in the sinonasal cavity, two in the trachea/bronchus, and one in the larynx. The study was approved by the local ethics committees.

\section{Immunohistochemistry}

Zinc formalin-fixed or neutral buffered formalinfixed paraffin-embedded tissue sections were cut and incubated with anti-MYB rabbit monoclonal antibody (clone EP769Y;1:200 dilution; Epitomics Inc., Burlingame, CA, USA) using a DAKO (Carpinteria, CA, USA) autostainer after pressure cooker antigen retrieval for $30 \mathrm{~s}$. The antibody recognizes a synthetic peptide corresponding to residues near the N-terminus of human MYB. The avidin-biotinimmunoperoxidase technique was used. Diaminobenzidine was the chromogen, and sections were counterstained with hematoxylin. MYB immunostaining was considered positive if greater than $5 \%$ of tumor cells displayed strong nuclear immunoreactivity. All cases were reviewed by three pathologists (LB, WK, HF).

\section{RT-PCR and Nucleotide Sequence Analyses}

Total RNA was extracted from 29 frozen adenoid cystic carcinoma tumor samples and normal salivary gland tissue (three cases), using the TRIzol reagent (Invitrogen, Carlsbad, CA, USA) and RNeasy Mini kit (Qiagen, Valencia, CA, USA). DNase-treated (DNA-free Ambion, Austin, TX, USA) total RNA was subsequently converted to cDNA, using the SuperScript First-Strand Synthesis System (Invitrogen) adenoid cystic carcinoma, according to the manufacturer's manual. Total RNA was also extracted from five $10 \mu \mathrm{m}$ sections obtained from paraffin blocks of 32 adenoid cystic carcinomas, and 18 non-adenoid cystic carcinoma neoplasms of the head and neck. The sections were deparaffinized in xylene, and RNA was isolated using the RNeasy FFPE kit (Qiagen). RNAs were subsequently converted to cDNA using the SuperScript FirstStrand Synthesis System (Invitrogen) with random hexamer primers, as recommended by the manufacturer. As controls for intact RNA and cDNA, RT-PCR reactions for expression of $A C T B$ ( $\beta$-actin) or $G A P D H$ were performed on all cDNAs.

The $M Y B-N F I B$ fusion transcripts were amplified by direct or nested PCR. Primer sequences for analysis of cDNAs prepared from frozen tumor tissues were as previously described. ${ }^{1}$ All cDNAs prepared from FFPE tumor material were screened for the most common $M Y B-N F I B$ fusion transcript variants, that is, $M Y B$ exon 14 fused to $N F I B$ exons 8c or 9, respectively. Touchdown PCR was performed using the $M Y B$ primer MYB-1910F-5'A GCTCCGTTTTAATGGCADENOID CYSTIC CAR CINOMA3' (located in exon 14) and the NFIB primer NFIB-1096R-5'GGGTATAAATGCCTGCCGTT3'(located in exon 8c), and direct PCR was performed using the
$M Y B$ primer MYB-1925F-5'GCACCAGCATCAGAA GATGA3' (located in exon 14) and the NFIB primer NFIB-1197R-5'CCGGTAAGATGGGTGTCCTA3' (located in exon 9). Tumors that were negative for these transcript variants were also analyzed for expression of chimeric transcripts consisting of $M Y B$ exon 12 fused to $N F I B$ exon 9, using the $M Y B$ primer MYB1693F-5'GCAGGATGTGATCAAACAGG3' (located in exon 12) and the NFIB primer NFIB-1197R5'CCGGTAAGATGGGTGTCCTA3' (located in exon 9). The $M Y B$ and $N F I B$ exons were numbered as described elsewhere. ${ }^{1}$ As positive control, adenoid cystic carcinomas with known $M Y B-N F I B$ fusion transcript variants were used. ${ }^{1}$ Each PCR reaction was repeated in triplicate. PCR products were gel-purified and sequenced using an ABI PRISM 310 Genetic Analyzer (Applied Biosystems, Foster City, CA, USA). The resulting sequences were analyzed using the BLAST tool provided by the National Center for Biotechnology Information (http://www.ncbi.nlm.nih.gov).

\section{Quantitative Real-Time PCR Analysis}

qPCR analysis was performed on frozen adenoid cystic carcinomas using the AB 7500 Fast Real-time PCR system (Applied Biosystems), as previously described. ${ }^{16} M Y B$ expression was analyzed using the TaqMan Gene Expression assay for $M Y B$ exons 1-2 (Hs00920554_m1) (Applied Biosystems). All samples were assayed in triplicate. The relative expression levels of $M Y B$ in tumor and normal salivary gland samples were calculated with SDS Software v2.0.1 (Applied Biosystems) using the comparative Ct method, ${ }^{17}$ with the housekeeping genes 18S (Hs99999901_s1) or GAPDH (Hs99999905-m1) as endogenous controls, and cDNA from normal salivary gland tissue as calibrator.

\section{Results}

Expression of $M Y B-N F I B$ and $M Y B$ Transcripts in Adenoid Cystic Carcinoma and Non-adenoid Cystic Carcinoma Tumors

To study the expression of the $M Y B-N F I B$ fusion in adenoid cystic carcinoma, we screened a series of 61 adenoid cystic carcinomas from different anatomic sites (29 frozen and 32 FFPE tumor samples), and 18 non-adenoid cystic carcinoma neoplasms of the head and neck by RT-PCR. The results are summarized in Table 2. Using primers located in $M Y B$ exons 5 , 6 or 12 , and in NFIB exon 9, we were able to identify one or more chimeric $M Y B-N F I B$ transcript variants in 25 of $29(86 \%)$ frozen adenoid cystic carcinoma tumor samples (Figure 1). Four cases were repeatedly negative for the $M Y B-N F I B$ fusion. Using primers located in $M Y B$ exons 12 or 14, and $N F I B$ exons 8c or 9, we detected chimeric $M Y B-N F I B$ 
Table $2 M Y B-N F I B$ fusion status in 61 adenoid cystic carcinomas from various anatomic sites, and in 18 non-adenoid cystic carcinoma neoplasms of the head and neck

\begin{tabular}{|c|c|c|c|c|}
\hline \multirow[t]{3}{*}{ Diagnosis } & \multicolumn{4}{|c|}{$\begin{array}{c}\text { MYB-NFIB fusion } \\
\text { status (by tissue } \\
\text { fixation) }\end{array}$} \\
\hline & \multicolumn{2}{|c|}{ FFPE } & \multicolumn{2}{|c|}{$F F$} \\
\hline & + & - & + & - \\
\hline Adenoid Cystic Carcinoma & 14 & 18 & 25 & 4 \\
\hline Salivary gland & 2 & 5 & 19 & 4 \\
\hline Breast & 2 & 2 & 0 & 0 \\
\hline Larynx & 1 & 3 & 1 & 0 \\
\hline Sinonasal cavity & 2 & 2 & 3 & 0 \\
\hline Trachea/Bronchus & 5 & 5 & 2 & 0 \\
\hline Vulva & 2 & 1 & 0 & 0 \\
\hline Non-adenoid cystic carcinoma & 0 & 18 & & \\
\hline Acinic cell carcinoma & 0 & 1 & & \\
\hline Basaloid squamous cell carcinoma & 0 & 6 & & \\
\hline Basal cell adenocarcinoma & 0 & 1 & & \\
\hline Epithelial-myoepithelial carcinoma & 0 & 1 & & \\
\hline Monomorphic adenoma & 0 & 4 & & \\
\hline Pleomorphic adenoma & 0 & 1 & & \\
\hline Polymorphous low-grade adenocarcinoma & 0 & 2 & & \\
\hline Salivary duct carcinoma & 0 & 2 & & \\
\hline
\end{tabular}

Abbreviations: FF, fresh frozen; FFPE, formalin-fixed paraffinembedded.
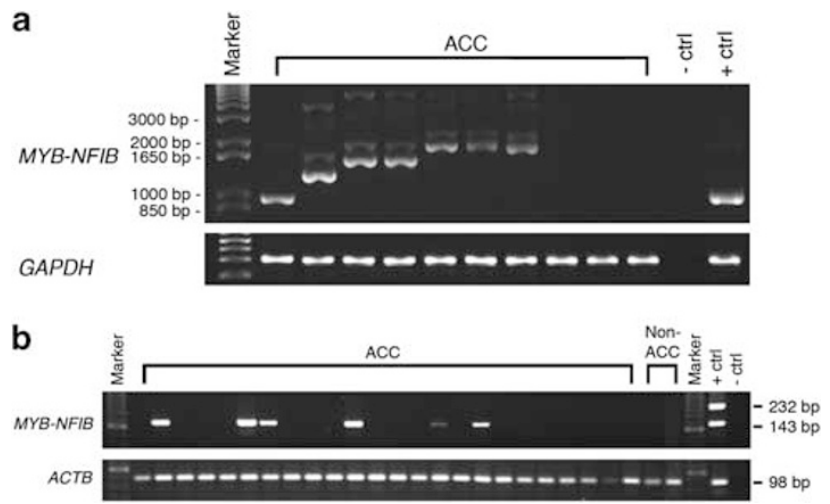

Figure 1 RT-PCR analyses of $M Y B-N F I B$ fusion transcripts in adenoid cystic carcinoma. (a) Detection of $M Y B-N F I B$ fusion transcripts in frozen tumor tissues from 10 adenoid cystic carcinomas, using primers located in $M Y B$ exon 5 and $N F I B$ exon 9 . Note that multiple bands corresponding to alternatively spliced $M Y B$ and/or NFIB exons are detected in several tumors. (b) Detection of $M Y B-N F I B$ fusion transcripts in FFPE tumor tissues from 24 adenoid cystic carcinomas, and two non-adenoid cystic carcinomas (acinic cell carcinoma and basal cell adenocarcinoma), using primers located in $M Y B$ exon 14 and $N F I B$ exon 9. Size markers, positive $(+)$ control (fusion-positive adenoid cystic carcinoma), and negative (-) control $\left(\mathrm{H}_{2} 0\right)$. GAPDH and $A C T B$ were used as internal controls to test for intact RNA and cDNA.

transcripts in 14 of 32 (44\%) FFPE adenoid cystic carcinoma tumor samples (data not shown). The identity of the chimeric transcript variants identified in both frozen and FFPE tumor samples was verified by nucleotide sequence analysis. The composition of the $M Y B-N F I B$ transcripts identified was in agreement with previously published data (not shown). In contrast, none of the 18 non-adenoid cystic carcinoma neoplasms of the head and neck expressed MYB-NFIB fusion transcripts (Table 2). Analysis of adenoid cystic carcinomas derived from various anatomic sites revealed that tumors from most sites were repeatedly fusion-positive, including the major and minor salivary glands (21/30), sinonasal cavity (5/7), trachea/bronchus (7/12), larynx $(2 / 5)$, breast $(2 / 4)$, and vulva $(2 / 3)$.

To study the frequency of $M Y B$ overexpression, we performed qPCR analysis of a series of 28 frozen adenoid cystic carcinoma samples from the aerodigestive tract, including 24 fusion-positive and four fusion-negative cases. As demonstrated in Figure 2, $M Y B$ was highly overexpressed in 25 of 28 (89\%) adenoid cystic carcinomas relative to normal salivary gland tissue. Of the 24 fusion-positive tumors, 22 had high $M Y B$ expression levels, and one of four fusion-negative tumors had low $M Y B$ expression levels. The remaining three fusion-negative adenoid cystic carcinomas had high $M Y B$ expression levels, comparable to those seen in the majority of fusionpositive tumors.

\section{Immunohistochemical Staining of MYB Protein in Adenoid Cystic Carcinoma and Non-adenoid Cystic Carcinoma Tumors}

The results of the immunohistochemical staining of MYB are summarized in Table 1, and illustrated in Figure 3a-e. Overall, 56 of $68(82 \%)$ adenoid cystic carcinomas stained positive for MYB (Figure 3a). There was no difference in MYB staining adenoid cystic carcinoma according to anatomic site of the tumors, or clinical features of the patients. The staining was entirely nuclear, and occurred in tubular, cribriform, and solid types. There was no difference in staining adenoid cystic carcinoma according to the grade of adenoid cystic carcinoma. However, in adenoid cystic carcinoma with tubular differentiation, the inner epithelial cells typically lacked MYB, whereas the outer myoepithelial cells were MYB positive (Figure 3b). In adenoid cystic carcinoma positive for MYB, the number of labeled cells varied, and, at times, the stained nuclei showed a peculiar zonal distribution in which the outer millimeter of tumor cell nuclei in tissue sections were stained, whereas the inner nuclei lacked positivity (Figure 3e). Also of note was the absence of MYB in areas of tumor necrosis (Figure 3c), which occurred in some non-adenoid cystic carcinoma neoplasms.

Of the non-adenoid cystic carcinoma tumors, 16 of $113(14 \%)$ were positive for MYB. These comprised four of five basaloid squamous cell carcinomas (Figure 3c), three of nine monomorphic adenomas/basal cell adenomas/salivary gland 


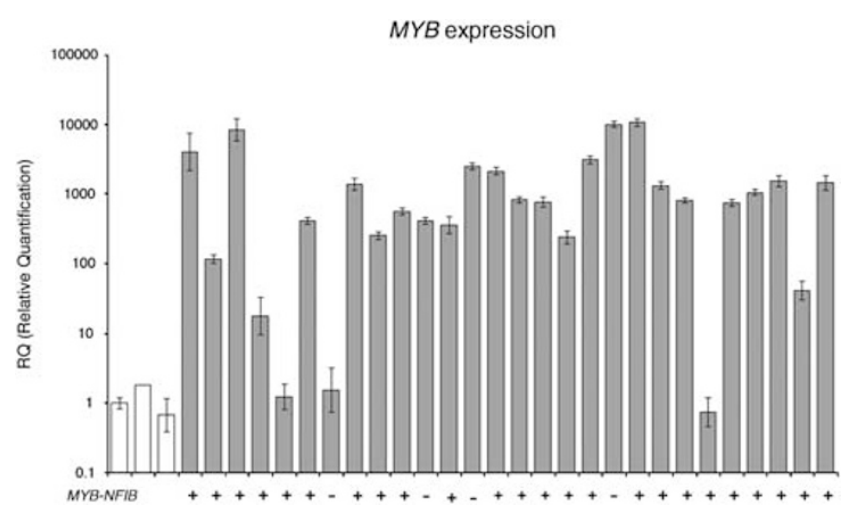

Figure 2 qPCR analysis of the expression of $M Y B$ in 28 fusionpositive $(+)$ and fusion-negative $(-)$ adenoid cystic carcinoma samples (grey bars), compared with normal salivary gland tissue (white bars).

adenoma, not otherwise specified (Figure 3d), two of 15 polymorphous low-grade adenocarcinomas, two of 18 salivary duct carcinomas, and one each of acinic cell carcinoma, basal cell adenocarcinoma, mucoepidermoid carcinoma, epithelial-myoepithelial carcinoma, and pleomorphic adenoma. RT-PCR analysis of MYB positive non-adenoid cystic carcinoma tumors revealed that they were negative for the MYB-NFIB fusion (Table 2). The sensitivity and specificity of MYB immunostaining for adenoid cystic carcinoma were 82 and $86 \%$, respectively $(P<0.001)$.

Scattered normal lymphocytes in various tissues were positive for MYB. In the breast, occasional normal myoepithelial cells and perilobular stromal cells labeled for the protein. MYB was not seen in acinar or ductal epithelium of normal salivary glands.

\section{Discussion}

In this investigation, we have used a combination of molecular and immunohistochemical methods to study the frequency of MYB-NFIB fusion and MYB expression in adenoid cystic carcinoma occurring at various anatomic sites. Although the overall frequency of $M Y B-N F I B$ fusion was $64 \%$ (39/61), we found a marked difference in the detection of the fusion transcript between our frozen $(86 \%)$ and FFPE $(44 \%)$ tissue specimens. We believe that the degradation and chemical cross-linking of RNA that occurs with formalin fixation and paraffin embedding likely resulted in the lower rate of $M Y B-N F I B$ fusion in the fixed samples. In addition, in these cases, we only tested for the most common $M Y B$ $N F I B$ transcript variants. In contrast, the $M Y B-N F I B$ fusion was not expressed in non-adenoid cystic carcinoma neoplasms of the head and neck, confirming the high specificity of the $M Y B-N F I B$ fusion for adenoid cystic carcinoma. ${ }^{1,2,14,15}$ Of note, no difference in MYB-NFIB fusion expression was observed between salivary gland and breast adenoid cystic carcinomas, despite their well-known difference in clinical behavior. ${ }^{18}$

Importantly, we also found that the $M Y B-N F I B$ fusion occured in adenoid cystic carcinomas from various anatomic sites. In addition to salivary gland, we found the fusion in adenoid cystic carcinomas located in the sinonasal cavity, tracheobronchial tree, larynx, breast, and vulva. These results, together with our previous observations, ${ }^{1}$ further support the notion that adenoid cystic carcinomas located in different anatomic sites not only have important morphologic features in common, but also chiefly evolve through activation of the same molecular pathways. The well-known difference in biological behavior between adenoid cystic carcinomas originating from different anatomic sites, for example salivary gland and breast, is thus not likely to be associated with the MYB-NFIB fusion, but is rather due to other currently unknown genetic alterations or to organ-specific differences in cellular milieu and anatomy.

The frequency of the MYB-NFIB fusion in salivary and non-salivary adenoid cystic carcinomas has varied in the earlier investigations. In our original study, ${ }^{1}$ we found the $M Y B-N F I B$ fusion in six of six adenoid cystic carcinomas with $\mathrm{t}(6 ; 9)$ translocations, and in an additional five cases from which cytogenetic data were not available (four of which were adenoid cystic carcinomas of the breast). Subsequently, Mitani et al ${ }^{14}$ found the $M Y B-N F I B$ fusion in a much lower frequency corresponding to approximately one-third of primary and metastatic salivary adenoid cystic carcinomas. More recently, West et al, ${ }^{15}$ using the method of fluorescence in situ hybridization, presented evidence indicating that $65 \%$ of salivary adenoid cystic carcinomas were positive for the MYB-NFIB fusion or had translocations of $M Y B$, not necessarily involving $N F I B$. Although the latter figure is in agreement with our finding of $M Y B-N F I B$ fusion positivity in $64 \%$ of salivary and non-salivary adenoid cystic carcinomas, the discrepancy seen in the detection of the fusion transcript between our frozen and FFPE tissue samples suggests that the true frequency of this genetic change in adenoid cystic carcinoma is higher. Although FFPE samples may not be as optimal as frozen specimens, ours is the first study demonstrating the utility of FFPE tumor material for RT-PCR analysis of the expression of $M Y B-N F I B$ in adenoid cystic carcinoma.

We also studied the frequency of $M Y B$ overexpression in both fusion-positive and fusionnegative adenoid cystic carcinomas using qPCR. Overall, $89 \%$ of the tumors overexpressed $M Y B$ RNA relative to normal salivary gland, including 22 of 24 fusion-positive tumors, and 3 of 4 fusionnegative tumors. These observations, together with recent data from two independent studies, ${ }^{14,15}$ further emphasize the significance of $M Y B$ as a major oncogene in the pathogenesis of adenoid 

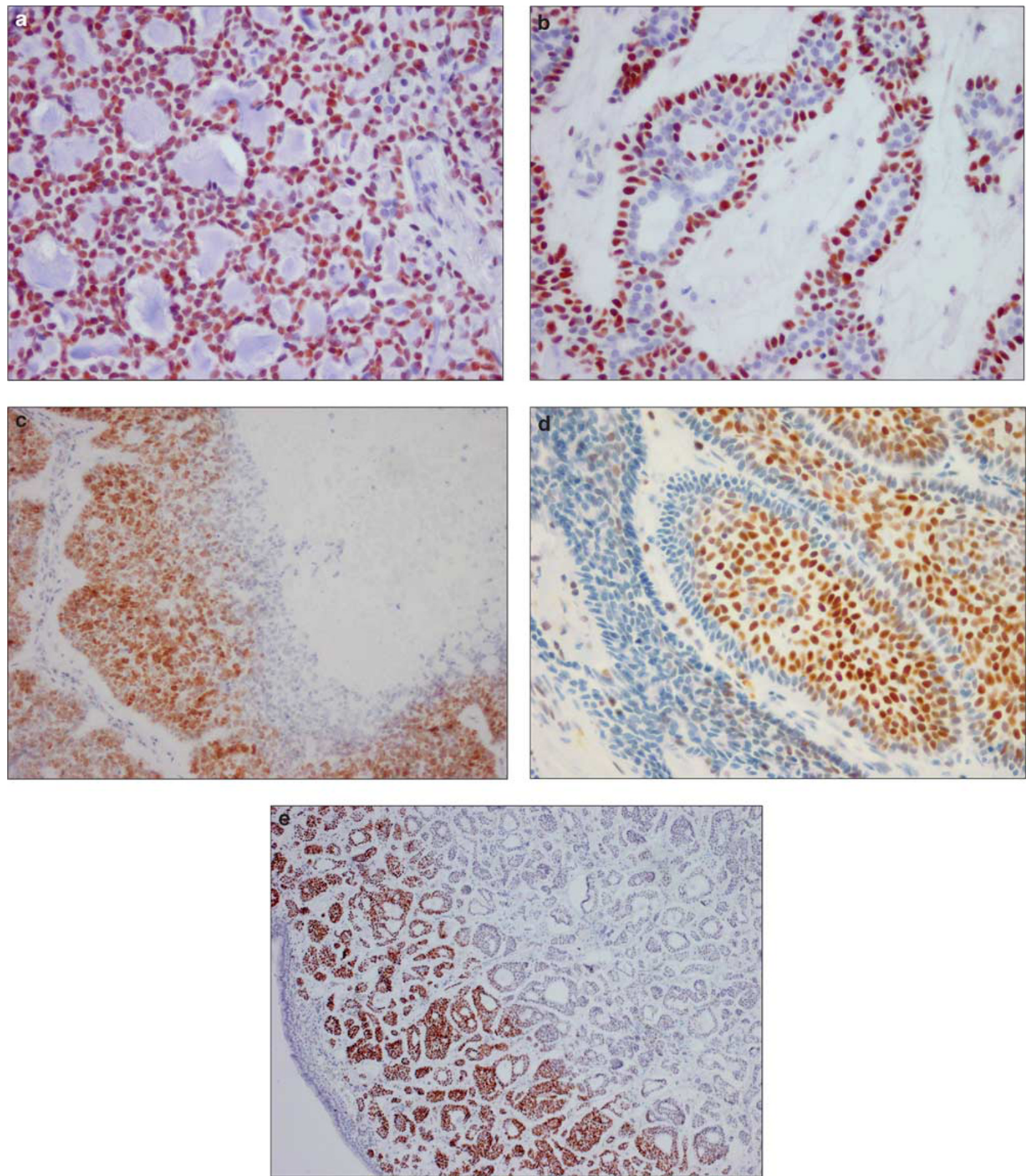

Figure 3 (a) Strong nuclear MYB immunoreactivity was typically observed in adenoid cystic carcinoma. (b) Staining was often restricted to non-luminal tumor cells in tubular foci. (c) This basaloid squamous cell carcinoma is positive for MYB, but staining is absent in areas of necrosis. (d) This monomorphic adenoma stains positive for MYB in some of the centers of tumor nests, whereas the peripheral palisades of cells are negative. (e) MYB immunostaining sometimes showed a peripheral pattern, presumably due to slower formalin fixation, and a short half-life of the MYB protein.

cystic carcinoma. The finding of high $M Y B$ expression levels in fusion-negative tumors also indicates that other mechanisms of $M Y B$ activation may exist. Whether these involve fusion partners other than
NFIB or represent novel mechanisms is currently unknown. However, preliminary RACE (rapid amplification of cDNA ends) analysis of a fusionnegative adenoid cystic carcinoma with high $M Y B$ 
expression failed to reveal a new $M Y B$ fusion partner (unpublished data). Our results also suggest that a subset of adenoid cystic carcinoma may be truly fusion-negative and/or do not overexpress $M Y B$. Detailed analysis of such non- $M Y B$-driven adenoid cystic carcinomas will be important in order to identify novel genes and mechanisms involved in the molecular pathogenesis of these neoplasms.

In our study, we found that 56/68 (82\%) of adenoid cystic carcinoma occurring at various anatomic sites stained positive for MYB protein. In the study by Mitani et al, ${ }^{14} 17$ of 20 (85\%) salivary adenoid cystic carcinoma that had the MYB-NFIB fusion stained positive for MYB, whereas 25 of 41 $(61 \%)$ fusion-negative adenoid cystic carcinoma also labeled for the protein. Using a different antibody from that used by us and Mitani et al, ${ }^{14}$ West et $a l^{15}$ found that $65 \%$ (24 of 37) of adenoid cystic carcinoma were strongly positive. It should be noted that we used a lower threshold for positivity than the investigators of these two earlier studies. It is of interest that in the tubular type of adenoid cystic carcinoma, the inner ductal layer of cells typically lacked MYB, whereas the outer myoepithelial layer showed strong MYB positivity. This finding was also observed by Mitani et al. ${ }^{14}$ The molecular mechanism for this staining pattern is unknown. The peculiar zonal staining pattern for MYB in some cases, in which positive nuclei were limited to the peripheral $1 \mathrm{~mm}$ of tissue sections, may indicate that MYB is very sensitive to formalin fixation and degrades quickly. It should be noted that normal MYB has a short half life of approximately $30 \mathrm{~min},{ }^{19}$ but the stability of the MYB-NFIB fusion protein is unknown.

Although the histopathologic diagnosis of the cribriform variant of adenoid cystic carcinoma does not usually pose a dilemma, there is some overlap of the tubular and solid variants with other neoplasms of the head and neck. Our study shows that analyses of the expression of MYB transcripts and/or protein by RT-PCR and immunohistochemistry would assist in the diagnosis of adenoid cystic carcinoma, but would not completely exclude other neoplasms in the differential diagnosis. In addition, a subset of adenoid cystic carcinomas are fusion-negative and lack MYB immunoreactivity, and there are also nonadenoid cystic carcinoma tumors that stain positive for MYB. However, as shown in this study, the latter cases are fusion-negative.

The $M Y B$ portion of the $M Y B-N F I B$ fusion has a deleted $3^{\prime}$-UTR, that normally contains the target sequences for miR-15a, miR-16, and miR-150, which negatively regulate MYB activity. ${ }^{20-23}$ This provides a constitutively active stimulus to genes variously involved in cell cycle regulation, apoptosis, angiogenesis, and adhesion. The normal $M Y B$ gene product is most prominently expressed in nonterminally differentiated cells, with decreasing expression observed in non-mitotically active cells. ${ }^{19,24}$ The murine MYB homolog has been shown to be highly expressed in embryonic salivary gland tissue. ${ }^{25}$ The normal NFIB gene product is a member of the NFI gene family, ${ }^{26,27}$ but its mechanistic importance, when it occurs as a partner to $M Y B$ in adenoid cystic carcinoma, is unknown. Interestingly, Stenman ${ }^{28}$ previously identified $N F I B$ as a fusion partner with HMGA2 in salivary pleomorphic adenomas with $\mathrm{t}(9 ; 12)$ translocations/insertions. ${ }^{27}$ The overall domain structure of the HMGA2-NFIB fusion resembles that of $M Y B-N F I B$ in that the C-terminal end of $N F I B$ is linked to the DNAbinding domains of the transcription factor $H M G A 2$, resulting in high expression levels of HMGA2-NFIB fusion transcript and protein. These findings raise the question of whether $N F I B$ in both fusion types may contribute stabilizing or regulatory elements to these transcription factors.

In summary, the present and previous studies demonstrate that $M Y B$ activation through gene fusion or other mechanisms is a major oncogenic event in tumors with typical adenoid cystic carcinoma histology, regardless of the site of tumor origin. In addition to being a new diagnostically useful biomarker for adenoid cystic carcinoma, MYB and its downstream effectors are also potential therapeutic targets. Future studies aiming at identifying the transcriptional targets of $M Y B-N F I B$ and $M Y B$ in adenoid cystic carcinoma will therefore be crucial in order to develop new potent therapies that may improve the survival of patients affected by this often fatal disease.

\section{Acknowledgements}

This study was supported in part by the Swedish Cancer Society, IngaBritt and Arne Lundbergs Research Foundation, BioCARE-a National Strategic Research Program at University of Gothenburg, and the Adenoid Cystic Carcinoma Research Foundation.

\section{Disclosure/conflict of interest}

The authors declare no conflict of interest.

\section{References}

1 Persson M, Andren Y, Mark J, et al. Recurrent fusion of MYB and NFIB transcription factor genes in carcinomas of the breast and head and neck. Proc Natl Acad Sci USA 2009;106:18740-18744.

2 Stenman G, Andersson MK, Andren Y. New tricks from an old oncogene: gene fusion and copy number alterations of MYB in human cancer. Cell Cycle 2010;9:2986-2995.

3 Nascimento AG, Amaral AL, Prado LA, et al. Adenoid cystic carcinoma of salivary glands. A study of 61 cases 
with clinicopathologic correlation. Cancer 1986;57: 312-319.

4 Barnes L, Eveson JW, Reichart P, et al. eds. World Health Organization Classification of Tumours. Pathology and Genetics of Head and Neck Tumours. IARC Press: Lyon, 2005, p 430.

5 Perzin KH, Gullane P, Clairmont AC. Adenoid cystic carcinomas arising in salivary glands: a correlation of histologic features and clinical course. Cancer 1978; 42:265-282.

6 Spiro RH, Huvos AG, Strong EW. Adenoid cystic carcinoma: factors influencing survival. Am J Surg 1979;138:579-583.

7 Azumi N, Battifora H. The cellular composition of adenoid cystic carcinoma. An immunohistochemical study. Cancer 1987;60:1589-1598.

8 Holst VA, Marshall CE, Moskaluk CA, et al. KIT protein expression and analysis of c-kit gene mutation in adenoid cystic carcinoma. Mod Pathol 1999;12: 956-960.

9 Penner CR, Folpe AL, Budnick SD. C-kit expression distinguishes salivary gland adenoid cystic carcinoma from polymorphous low-grade adenocarcinoma. Mod Pathol 2002;15:687-691.

10 Stenman G, Sandros J, Dahlenfors R, et al. 6q- and loss of the $\mathrm{Y}$ chromosome-two common deviations in malignant human salivary gland tumors. Cancer Genet Cytogenet 1986;22:283-293.

11 Higashi K, Jin Y, Johansson M, et al. Rearrangement of 9p13 as the primary chromosomal aberration in adenoid cystic carcinoma of the respiratory tract. Genes Chromosomes Cancer 1991;3:21-23.

12 Nordkvist A, Mark J, Gustafsson H, et al. Non-random chromosome rearrangements in adenoid cystic carcinoma of the salivary glands. Genes Chromosomes Cancer 1994;10:115-121.

13 Mitelman F, Johansson B, Mertens F, eds. Mitelman Database of Chromosome Aberrations and Gene Fusions in Cancer. http://cgap.nci.nih.gov/Chromo somes/Mitelman 2011.

14 Mitani Y, Li J, Rao PH, et al. Comprehensive analysis of the MYB-NFIB gene fusion in salivary adenoid cystic carcinoma: incidence, variability and clinicopathological significance. Clin Cancer Res 2010;16:4722-4731.

15 West RB, Kong C, Clarke N, et al. MYB expression and translocation in adenoid cystic carcinoma and other salivary gland tumors with clinicopathologic correlation. Am J Surg Pathol 2011;35:92-99.
16 Persson F, Winnes M, Andrén Y, et al. High-resolution array CGH analysis of salivary gland tumors reveals fusion and amplification of the FGFR1 and PLAG1 genes in ring chromosomes. Oncogene 2008;27: 3072-3080.

17 Livak KJ, Schmittgen TD. Analysis of relative gene expression data using real-time quantitative PCR and the 2(-DD C(T)) method. Methods 2001;25: 402-408.

18 Marchio C, Weigelt B, Reis-Filho JS. Adenoid cystic carcinomas of the breast and salivary glands (or 'The strange case of Dr Jekyll and Mr Hyde' of exocrine gland carcinomas). J Clin Pathol 2010;63: 220-228.

19 Ramsay RG, Gonda TJ. MYB function in normal and cancer cells. Nat Rev Cancer 2008;8:523-534.

20 Xiao C, Calando DP, Galler G, et al. MiR-150 controls B cell differentiation by targeting the transcription factor c-Myb. Cell 2007;131:146-159.

21 Chung EY, Dews M, Cozma D, et al. c-Myb oncoprotein is an essential target of the dleu2 tumor suppressor microRNA cluster. Cancer Biol Ther 2008;7: 1758-1764.

22 Lin YC, Kuo MW, Yu J, et al. c-Myb is an evolutionary conserved miR-150 target and miR-150/c-Myb interaction is important for embryonic development. Mol Biol Evol 2008;25:2189-2198.

23 Zhao H, Kalota A, Jin S, et al. The c-myb protooncogene and microRNA-15a comprise an active autoregulatory feedback loop in human hematopoietic cells. Blood 2009;113:505-516.

$24 \mathrm{Oh} \mathrm{IH}$, Reddy EP. The myb gene family in cell growth, differentiation and apoptosis. Oncogene 1999;18: 3017-3033.

25 Visel A, Thaller C, Eichele G. GenePaint.org: an atlas of gene expression patterns in the mouse embryo. Nucleic Acids Res 2004;32:D552-D556.

26 Qian F, Kruse U, Lichter $\mathrm{P}$, et al. Chromosomal localization of the four genes (NFIA, B, C, and X) for the human transcription factor nuclear factor I by FISH. Genomics 1995;28:66-73.

27 Geurts JM, Schoenmakers EF, Roijer E, et al. Identification of NFIB as recurrent translocation partner gene of HMGIC in pleomorphic adenomas. Oncogene 1998; 16:865-872.

28 Stenman G. Fusion oncogenes and tumor type specificity-insights from salivary gland tumors. Semin Cancer Biol 2005;15:224-235. 